\title{
Orbital features of distant trans-Neptunian objects induced by giant gaseous clumps
}

\begin{abstract}
V. V. Emel'yanenko
Institute of Astronomy, Russian Academy of Sciences, Moscow 119017, Russia

e-mail: vvemel@inasan.ru

Received 22 July 2020 / Accepted 6 October 2020

ABSTRACT

Context. The discovery of distant trans-Neptunian objects has led to heated discussions about the structure of the outer Solar System. Aims. We study the dynamical evolution of small bodies from the Hill regions of migrating giant gaseous clumps that form in the outer solar nebula via gravitational fragmentation. We attempt to determine whether the observed features of the orbital distribution of distant trans-Neptunian objects could be caused by this process.

Methods. We consider a simple model that includes the Sun, two point-like giant clumps with masses of $\sim 10$ Jupiter masses, and a set of massless objects initially located in the Hill regions of these clumps. We carry out numerical simulations of the motions of small bodies under gravitational perturbations from two giant clumps that move in elliptical orbits and approach each other. The orbital distribution of these small bodies is compared with the observed distribution of distant trans-Neptunian objects.

Results. In addition to the known grouping in longitudes of perihelion, we note new features for observed distant trans-Neptunian objects. The observed orbital distribution points to the existence of two groups of distant trans-Neptunian objects with different dynamical characteristics. We show that the main features of the orbital distribution of distant trans-Neptunian objects can be explained by their origin in the Hill regions of migrating giant gaseous clumps. Small bodies are ejected from the Hill regions when the giant clumps move in high-eccentricity orbits and have a close encounter with each other.

Conclusions. The resulting orbital distribution of small bodies in our model and the observed distribution of distant trans-Neptunian objects have similar features.
\end{abstract}

Key words. Kuiper belt: general - planet-disk interactions - methods: numerical

\section{Introduction}

The discovery of distant trans-Neptunian objects (TNOs) moving in orbits with semimajor axes $a>150$ au and perihelion distances $q>30$ au has provided unexpected information about the structure of the outer Solar System. Trujillo \& Sheppard (2014) first suggested that there is a concentration of arguments of perihelion $\omega$ near the value $\omega=0^{\circ}$. This value corresponds to one of the centers of the libration zones in the von Zeipel-LidovKozai mechanism of secular perturbations (Von Zeipel 1910; Lidov 1962; Kozai 1962). Therefore, an assumption was made about the existence of a distant planet producing this effect. Later, Batygin \& Brown (2016) noted that, to a greater extent, there is a grouping of longitudes of ascending nodes $\Omega$ and longitudes of perihelion $\pi=\omega+\Omega$, and this effect is associated with the combined action of orbital and secular resonances (Batygin \& Morbidelli 2017). Although Lawler et al. (2017) and Shankman et al. (2017a) scrutinized observational bias in the Outer Solar System Origins Survey sample and concluded that there is no evidence of clustering in angular orbital element distributions (see also Bernardinelli et al. 2020; Clement \& Kaib 2020; Trujillo 2002), Brown (2017) estimated a rather low probability for the observed sample of TNOs with $a>230$ au to be drawn from a uniform population. Detailed studies have shown that the observed features in the distribution of angular elements for distant TNOs could be produced by a planet with a mass of $\sim 5-10$ Earth masses moving in an orbit with a semimajor axis $a \sim 400-800 \mathrm{au}$, eccentricity $e \sim 0.2-0.5$, and inclination $i \sim 15-25^{\circ}$ (Batygin \& Brown 2016; Batygin et al. 2019).

Although the dynamical arguments seem quite convincing (Batygin \& Morbidelli 2017), the question of the actual existence of a ninth planet in the Solar System remains open. Despite ongoing searches, the planet has not yet been discovered. An explanation for the formation of such a massive and distant planet is also difficult to establish (Batygin et al. 2019). Shankman et al. (2017b) and Kavelaars et al. (2020) have found dynamical effects that a massive distant planet would have on the distant TNO population that were not highlighted in the initial published theory of Batygin \& Brown (2016). Nesvorný et al. (2017) concluded that the inclination distribution of Jupiterfamily comets is wider than the observed one in models with a ninth planet.

However, without existing additional perturbers, it is difficult to explain the observed clustering of the apsidal lines of distant TNOs. It has been suggested that a self-gravitating massive disk of TNOs, and not a ninth planet, may be sustaining the confinement of the longitudes of perihelion (Sefilian \& Touma 2019; Zderic et al. 2020). This suggestion was criticized in Batygin et al. (2019). Their main objections concern the lack of material needed for the formation and existence of the massive disk of TNOs. The arguments discussed were based on the widespread "core accretion" theory of planet formation (e.g., 
Safronov 1991; Montmerle et al. 2006; Armitage 2018) and the classical "minimum mass solar nebula" model of the solar protoplanetary disk (e.g., Weidenschilling 1977; Hayashi 1981; Desch 2007; Crida 2009).

An alternative planet formation scenario is provided by the "gravitational instability" theory (see, for example, the reviews Kratter \& Lodato 2016; Nayakshin 2017). The detection of exoplanets in wide orbits provides observational support for this theoretical scenario (e.g., Marois et al. 2008; Bohn et al. 2020). The gravitational instability of protoplanetary disks is envisioned to lead to the formation of self-gravitating clouds of gas and dust (the canonical gas-to-dust mass ratio is 100:1), called giant gaseous clumps. Recently, it has been shown that such clumps participate in a complex dynamical interaction with the disk, which notably leads to the migration of clumps (Mayer et al. 2002; Vorobyov \& Basu 2005; Nayakshin 2010; Baruteau et al. 2011; Zhu et al. 2012; Stamatellos 2015; Vorobyov \& Elbakyan 2018). Moreover, migrating clumps can experience close encounters with each other, which often lead to the ejection of objects into hyperbolic orbits (Terquem \& Papaloizou 2002; Vorobyov \& Elbakyan 2018). Emel'yanenko (2020) showed that the observed distribution of angular orbital elements of TNOs could be created by the perturbations of such clumps, based on the results of hydrodynamical simulations in Vorobyov \& Elbakyan (2018).

In this Letter, we first discuss new features of the observed distant TNOs. Then we consider dynamical processes of migration and close encounters of giant gaseous clumps, motivated by the results of Vorobyov \& Elbakyan (2018). We attempt to determine whether the observed orbital distribution of distant TNOs could be induced by such clumps moving in orbits with different inclinations to the ecliptic. For this purpose, we study the dynamical evolution of small bodies from the Hill regions of the giant clumps. In the numerical hydrodynamics simulations of Vorobyov \& Elbakyan (2018), the first clump forms $60 \mathrm{kyr}$ after the star formation, and no clumps are present in the protoplanetary disk at $0.5 \mathrm{Myr}$. Thus, our model relates to a very early stage in the evolution of the protoplanetary disk when the present-day planets were not yet formed.

\section{Orbital features of the observed distant trans-Neptunian objects}

We consider TNOs with $q>40 \mathrm{au}$, in contrast to many previous works (e.g., Trujillo \& Sheppard 2014; Batygin \& Brown 2016) that analyzed orbits with $q>30 \mathrm{au}$. This restriction seems more justified when studying dynamical mechanisms in the early stages of Solar System formation. Orbits with perihelia near the orbit of Neptune have changed significantly over the lifetime of the Solar System due to planetary perturbations. Moreover, the stronger restriction on perihelion distances allows us to take into consideration not only orbits with very large $a$, but also a population of detached TNOs with smaller $a$. The heliocentric orbital elements presented on the website of the Minor Planet Center ${ }^{1}$ on January 16, 2020, were used for objects observed in at least two oppositions. A list of these objects and their orbital elements are given in Appendix A.

Figure 1 shows the distribution of semimajor axes and inclinations for TNOs with $q>40$ au and $a>60 \mathrm{au}$. All the objects with $a<160$ au have $i>20^{\circ}$. Large inclinations are not very unusual because there are dynamical mechanisms, in particular resonances, by which TNOs can reach high-inclination orbits.

\footnotetext{
1 https://wWW.minorplanetcenter.net/iau/lists/
}

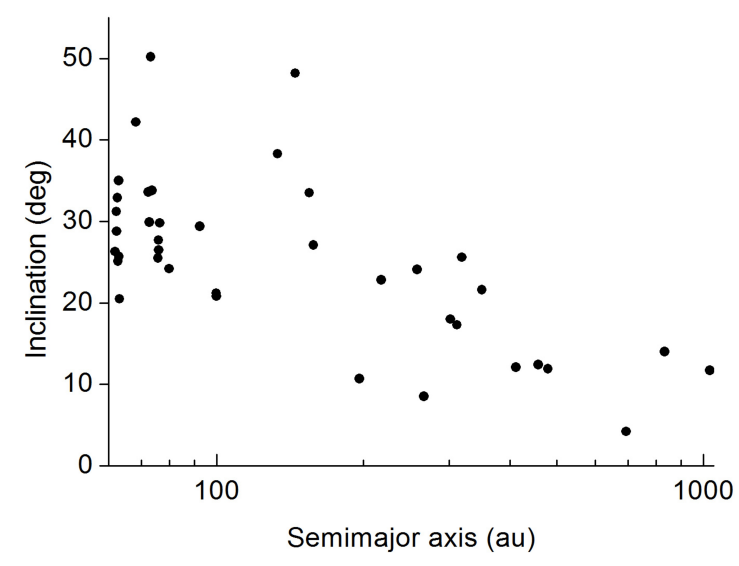

Fig. 1. Distribution of semimajor axes and inclinations for observed multiple-opposition TNOs with $q>40$ au and $a>60$ au.

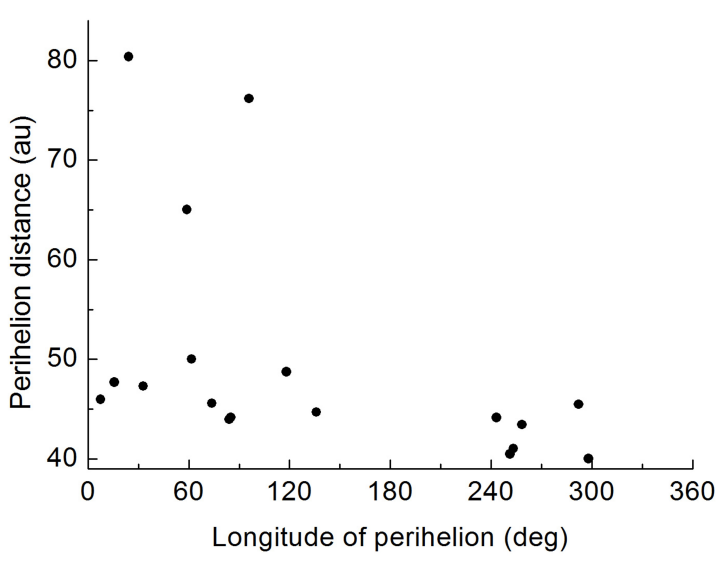

Fig. 2. Distribution of longitudes of perihelion and perihelion distances for observed multiple-opposition TNOs with $q>40$ au and $a>100$ au.

Really surprising is the lack of low-inclination orbits for all objects with $q>40$ au and $a>60$ au. Objects with larger semimajor axes have lower inclinations on average, and all the objects with $a>400$ au have $i<15^{\circ}$. Figure 2 shows the distribution of longitudes of perihelion and perihelion distances for TNOs with $q>40$ au and $a>100$ au. For $q>40$ au, two concentrations of longitudes of perihelion are clearly visible, even for objects with $a>100 \mathrm{au}$. The first group of 12 objects has the mean value $\bar{\pi}=66^{\circ}$, and the second group of six objects has the mean value $\bar{\pi}=266^{\circ}$. The observed values of perihelion distances for these groups are different. For the first group, perihelion distances extend from 44 au to $80 \mathrm{au}$. Perihelion distances for the second group are smaller; they do not exceed $45 \mathrm{au}$. The orbits in these groups also differ in regards to the distribution of inclinations. While the inclinations for the first group are in the interval $\left(4^{\circ}, 20^{\circ}\right)$ with the mean value $\bar{i}=15^{\circ}$, the inclinations for the second group spread from $14^{\circ}$ to $48^{\circ}$ with the mean value $\bar{i}=30^{\circ}$. The described features give us reason to suppose that we are observing two groups of objects with different dynamical characteristics. In this Letter, we assume that these features are inherent in the real distribution of distant TNOs and are not generated by observational biases.

\section{Methods}

We study a system of two interacting gaseous clumps surrounded by planetesimals in the Hill regions defined by the radius 
$R_{\mathrm{H}} \approx r_{\mathrm{h}}\left((m / 3)^{1 / 3}-\frac{1}{3}(m / 3)^{2 / 3}-\frac{1}{9}(m / 3)\right)$, where $r_{\mathrm{h}}$ is the clump heliocentric distance and $m$ is the clump mass in the astronomical system of units (e.g., Murray \& Dermott 1999). This approach is based on the results from Vorobyov \& Elbakyan (2018) regarding the dynamics of clumps. In this model, the outer object with a lower mass moves in an initial orbit with $e \sim 0.5$. The inner object starts moving in a near-circular orbit with $a \sim 100 \mathrm{au}$. After a certain period of time, the objects approach each other. As a result of mutual perturbations, these objects enter into orbits with high eccentricities. The outer object is ejected to a near-parabolic or hyperbolic orbit. The inner object enters an orbit with $q \sim 30 \mathrm{au}$, and this orbit is quickly rounded off at such a distance due to interaction with the protoplanetary gas disk (see Fig. 17 in Vorobyov \& Elbakyan 2018).

We assume that planetesimals exist in the Hill regions of giant clumps during their migration stage. According to the calculations in Vorobyov \& Elbakyan (2018), the lifetimes of giant clumps in the protoplanetary disk do not exceed $0.5 \mathrm{Myr}$. Although the question of the formation of small bodies in such a short time period is open, there are many works that consider a very rapid formation of small bodies in the outer Solar System. For example, Wahlberg Jansson \& Johansen (2014) claim that planetesimals of various sizes can be made at 40 au in their numerical experiments through the collapse of pebble clouds formed by the streaming instability in protoplanetary disks: more than $\sim 100 \mathrm{~km}$ in $\sim 25$ years, $10 \mathrm{~km}$ in several hundred years, and $1 \mathrm{~km}$ in several thousand years. Boley et al. (2010), Nayakshin \& Cha (2012), and Nayakshin (2017) argue that making solid bodies is more straightforward inside the giant gaseous clump regions than in circumstellar disks.

The main dynamical mechanism of creating the population of small bodies moving in distant heliocentric orbits is associated with a decrease in the size of the Hill region when the clump moves from aphelion to perihelion. Some bodies formed in the Hill region are outside this region at a certain time and are ejected from the vicinity of the clump.

Here we consider simple models in which the clump is treated as a point mass and the planetesimals are treated as massless particles. The particles are initially placed in the plane of the heliocentric motion of the clump. The planetesimal disk has the initial surface density profile of $\Sigma(r) \propto 1 / \sqrt{1+\left(r / r_{0}\right)^{4}}$ (Vorobyov \& Elbakyan 2019), where $r$ is the radius of a circular orbit around the center of the clump. According to the numerical simulations in Vorobyov \& Elbakyan (2019), $r_{0}$ is usually much less than $R$, where $R$ is the clump radius; then, $\Sigma(r)$ is approximately proportional to $r^{-2}$ for $r>R$. For each $r$, the initial particles have a random uniform distribution along the orbit. We consider particles with initial $r>R$ and prograde Keplerian velocities.

The integration of the equations of motion is based on the symplectic integrator for the $N$-body problem (Emel'yanenko 2007). To describe the changes of orbits during the migration of clumps, we applied a method used in Emel'yanenko (2011).

\section{Numerical simulations}

We consider a simple model that includes the Sun, two pointlike massive clumps, and a set of particles initially located in the Hill regions of these clumps. To model the motion of clumps, we relied on the results of Vorobyov \& Elbakyan (2018), but we carried out numerical simulations with different masses and orbits of clumps. Below we demonstrate variants for which the basic features of the simulated distribution are similar to those

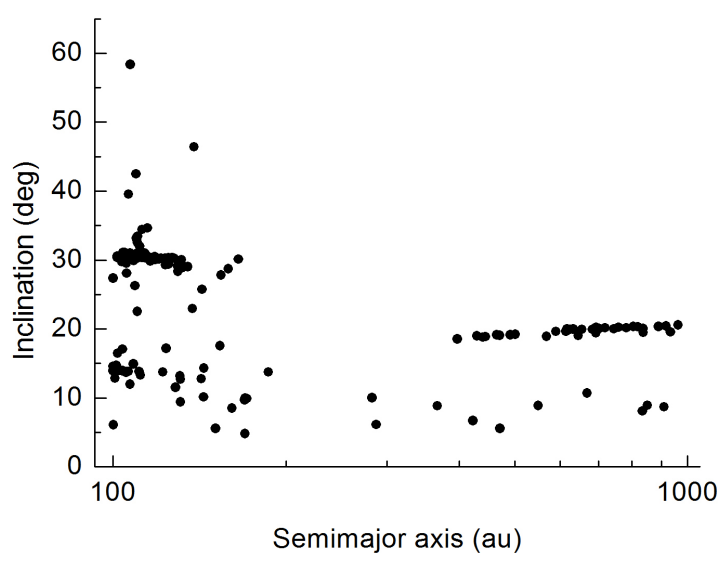

Fig. 3. Distribution of semimajor axes and inclinations for simulated particles with $40<q<80$ au and $100<a<1000 \mathrm{au}$.

observed for distant TNOs. All the analyzed orbital elements are heliocentric.

In the first stage of our model, two clumps migrate slightly inward. The outer clump, with a mass of 12 Jupiter masses and radius $R=6.4 \mathrm{au}$, starts in the aphelion of the orbit with $a=187 \mathrm{au}, q=123 \mathrm{au}, i=30^{\circ}$, and $\pi=257^{\circ}$. The inner object, with a mass of 17 Jupiter masses and radius $R=7.2 \mathrm{au}$, starts in the orbit with $a=110 \mathrm{au}, q=100 \mathrm{au}, i=15^{\circ}$, and $\pi=98^{\circ}$. Near every clump, 1000 particles are initially distributed between $R$ and $R_{\mathrm{H}}$ at the aphelion distances of the clumps (37 au for the outer clump and 20 au for the inner clump). The dynamical evolution of the clumps is shown in Fig. B.1. The orbital distribution of particles in the first stage (after 2300 years) is described in Appendix C.

In our model, there is then a close encounter of the clumps. This encounter occurs near the perihelion of the outer clump and the aphelion of the inner clump. As a result, the inner clump evolves to have an orbit with $q=37 \mathrm{au}$. According to Vorobyov \& Elbakyan (2018), the orbit quickly becomes nearcircular at such a distance due to interaction with the protoplanetary gas disk, and the clump loses its mass. After that, this object weakly disturbs distant particles. Therefore, we consider the orbital distribution of particles near the moment of the perihelion passage of the inner clump.

Figure 3 shows the distribution of semimajor axes and inclinations for particles with $40<q<80$ au and $100<a<1000$ au (the observable region of distant TNOs). Only particles located outside the Hill regions of the clumps are included. Figure 4 shows the distribution of longitudes of perihelion and perihelion distances for the same particles. The main features of these distributions are consistent with those shown in Figs. 1 and 2. On average, the inclinations of the orbits decrease as the semimajor axes increase. There are two concentrations of particles in the distribution of longitudes of perihelion (applying the Kolmogorov-Smirnov statistical test to those presented in Fig. 4 shows that the hypothesis of a uniform distribution is rejected with a probability of more than 0.9999). We note that orbits of these two groups differ in inclinations (for example, the mean inclination of orbits with $\pi<180^{\circ}$ is equal to $16.4^{\circ}$, while the mean inclination of orbits with $\pi>180^{\circ}$ is equal to $28.7^{\circ}$ ).

\section{Discussion}

We have studied a model in which two giant gaseous clumps approach each other and move into high-eccentricity orbits. In 


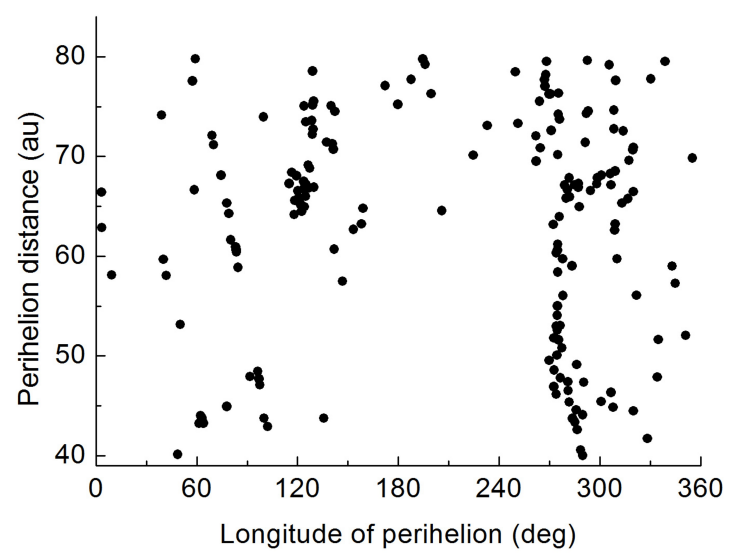

Fig. 4. Distribution of longitudes of perihelion and perihelion distances for simulated particles with $40<q<80$ au and $100<a<1000 \mathrm{au}$.

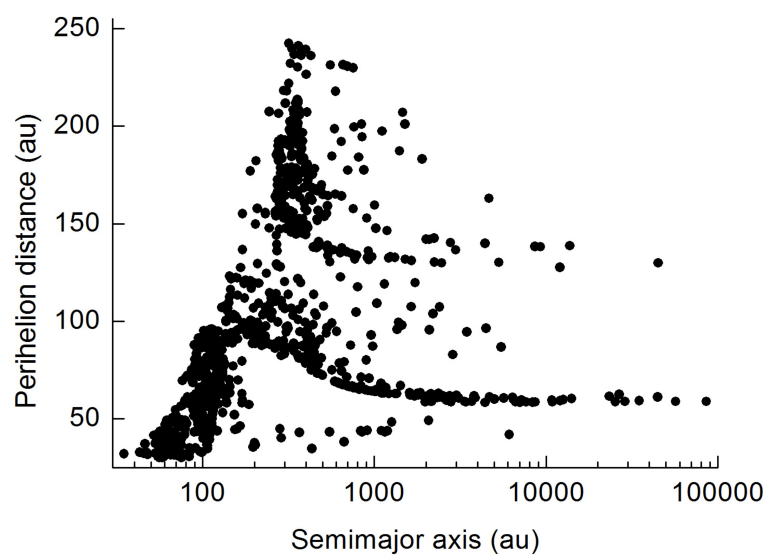

Fig. 5. Distribution of semimajor axes and perihelion distances for simulated particles with $q>30$ au and positive $a<10^{5}$ au.

this Letter, we do not consider the possible further evolution of such clumps. According to Vorobyov \& Elbakyan (2018), the inner clump is rapidly destroyed due to tidal effects. In the model discussed above, the final orbit of the outer object has $q=106 \mathrm{au}$ and $a=544 \mathrm{au}$. It is natural to assume that this clump also gradually disperses in such an orbit, adding to the swarm of solid bodies in the distant Solar System. In some variants of our simulations, such a clump is ejected into a hyperbolic orbit, as in the work of Vorobyov \& Elbakyan (2018).

The clustering of orbital elements is clearly discernible in our simulations. But this clustering should be sustained by some mechanism during the lifetime of the Solar System in order to be seen in the present observational data. For example, planetary perturbations are large enough to disperse the initial clustering of longitudes of perihelion throughout the lifetime of the Solar System, even for Sedna-type objects (e.g., Saillenfest et al. 2019). Sefilian \& Touma (2019) propose that a self-gravitating disk of TNOs can maintain its orbital features throughout the lifetime of the Solar System. This scenario is very relevant for our model. Figure 5 shows the distribution of semimajor axes and perihelion distances for all particles with $q>30$ au and positive $a<10^{5}$ au. Obviously, it is not difficult to obtain the required mass of the disk of $\sim 10$ Earth masses (Batygin et al. 2019; Sefilian \& Touma 2019) if the initial giant clumps have masses of $\sim 10$ Jupiter masses. We leave a detailed study of the orbital evolution of the disk population over the lifetime of the Solar System to a future paper.
It is difficult to discuss in more detail the consistency of the suggested dynamical process and the observed distribution of distant TNOs. On the one hand, the number of detected distant TNOs is currently too small. On the other hand, it is evident that the process of the formation and migration of giant gaseous clumps is much more complicated than in our model. In particular, while we consider only two clumps on a relatively short timescale, numerical hydrodynamics simulations (e.g., Vorobyov 2013; Vorobyov \& Pavlyuchenkov 2017; Vorobyov \& Elbakyan 2018) show that the formation process of giant gaseous clumps continues for $\sim 0.5 \mathrm{Myr}$, with many clumps involved. It is actually surprising that the studied simple model describes the main features of the observed orbital distribution of distant TNOs so well.

In addition to the abovementioned features, Fig. 5 shows that there is a gap in the distribution of perihelion distances in the observable region for $a>170$ au. A similar gap exists for the observed distant TNOs (although there are also no observed TNOs with $q>56$ au for smaller semimajor axes). This figure also shows that our model produces many objects with $30<q<40$ au and high eccentricities. Although modern theories relate the origin of such objects to the result of gravitational scattering by Neptune (the so-called scattered disk) (e.g., Morbidelli \& Nesvorný 2020), our numerical simulations demonstrate that giant clumps migrating in the outer Solar System could play a significant role in creating this population of TNOs.

Future observations confirming the proposed dynamical process would have important implications for understanding the structure of the outer Solar System. We would like to note three points that were not explicitly discussed in the Letter but follow from the general approach of the considered model.

First, the solar protoplanetary disk was more extended than the classical minimum mass solar nebula model formulates. The theory of a massive, extended solar nebula has long existed, and it is supported by observations of massive disks around pre-main sequence and main sequence stars (e.g., Bailey 1994).

Second, there are many undiscovered small bodies with $q>$ $80 \mathrm{au}$ (see Fig. 5). A significant additional contribution can come from the disintegration of the outer clump.

Thirdly, Fig. 5 shows that many objects in our model reach high-eccentricity orbits located in both the inner core $\left(10^{3}<\right.$ $a<10^{4} \mathrm{au}$ ) (Hills 1981) and the outer component $\left(a>10^{4} \mathrm{au}\right)$ of the Oort cloud (Oort 1950). Therefore, the formation of the Oort cloud may be associated with the described process, although the main models relate the origin of the Oort cloud to the scattering of remaining planetesimals by the formed planets (e.g., Fernández 1980; Duncan et al. 1987; Dones et al. 2004; Emel'yanenko et al. 2007).

\section{Conclusions}

We have analyzed the orbital distribution of distant TNOs. We note several new features of the observed objects in addition to their known groupings in longitudes of perihelion. Although the number of detected objects is still too small to make final conclusions, the observed orbital distribution points to the existence of two groups of distant TNOs with different dynamical characteristics.

We have shown that the main features of the observed orbital distribution of distant TNOs can be explained by their origin in the Hill regions of migrating giant gaseous clumps that form in the outer solar nebula via gravitational fragmentation. In our model, two clumps with masses of $\sim 10$ Jupiter masses 
interact gravitationally with the small bodies that surround them The small bodies are ejected from the Hill regions when two giant clumps move in high-eccentricity orbits and have a close encounter with each other. The resulting orbital distribution of small bodies in our model and the observed distribution of distant TNOs have similar features.

Acknowledgements. The author acknowledges the support of the Large Scientific Project of the Russian Ministry of Science and Higher Education "Theoretical and experimental studies of the formation and evolution of extrasolar planetary systems and characteristics of exoplanets" (project No. 13.1902.21.0039). The calculations were carried out using the MVS-100K supercomputer of the Joint Supercomputer Center of the Russian Academy of Sciences. The author thanks an anonymous referee for helpful comments.

\section{References}

Armitage, P. J. 2018, in Handbook of Exoplanets, eds. H. J. Deeg, \& J. A Belmonte, 135

Bailey, M. E. 1994, in Asteroids, Comets, Meteors 1993: Proceedings of the 160th Symposium of the International Astronomical Union, eds. A. Milani, M. Di Martino, \& A. Cellino, 443

Baruteau, C., Meru, F., \& Paardekooper, S.-J. 2011, MNRAS, 416, 1971

Batygin, K., \& Brown, M. E. 2016, AJ, 151, 22

Batygin, K., \& Morbidelli, A. 2017, AJ, 154, 229

Batygin, K., Adams, F. C., Brown, M. E., \& Becker, J. C. 2019, Phys. Rep., 805,

Bernardinelli, P. H., Bernstein, G. M., Sako, M., et al. 2020, Planet. Sci. J., 1, 28 Bohn, A. J., Kenworthy, M. A., Ginski, C., et al. 2020, ApJ, 898, L16

Boley, A. C., Hayfield, T., Mayer, L., \& Durisen, R. H. 2010, Icarus, 207, 509 Brown, M. E. 2017, AJ, 154, 65

Clement, M. S., \& Kaib, N. A. 2020, AJ, 159, 285

Crida, A. 2009, ApJ, 698, 606

Desch, S. J. 2007, ApJ, 671, 878

Dones, L., Weissman, P. R., Levison, H. F., \& Duncan, M. J. 2004, in Comets II, eds. M. C. Festou, H. U. Keller, \& H. A. Weaver, 153

Duncan, M., Quinn, T., \& Tremaine, S. 1987, AJ, 94, 1330

Emel'yanenko, V. V. 2007, Celest. Mech. Dyn. Astron., 98, 191

Emel'yanenko, V. V. 2011, Sol. Syst. Res., 45, 402

Emel'yanenko, V. V. 2020, Sol. Syst. Res., 54, 64

Emel'yanenko, V. V., Asher, D. J., \& Bailey, M. E. 2007, MNRAS, 381, 779
Fernández, J. A. 1980, Icarus, 42, 406

Hayashi, C. 1981, Prog. Theor. Phys. Suppl., 70, 35

Hills, J. G. 1981, AJ, 86, 1730

Kavelaars, J. J., Lawler, S. M., Bannister, M. T., \& Shankman, C. 2020, in The

Trans-Neptunian Solar System, eds. D. Prialnik, M. A. Barucci, \& L. Young, 61

Kozai, Y. 1962, AJ, 67, 591

Kratter, K., \& Lodato, G. 2016, ARA\&A, 54, 271

Lawler, S. M., Shankman, C., Kaib, N., et al. 2017, AJ, 153, 33

Lidov, M. L. 1962, Planet. Space Sci., 9, 719

Marois, C., Macintosh, B., Barman, T., et al. 2008, Sci, 322, 1348

Mayer, L., Quinn, T., Wadsley, J., \& Stadel, J. 2002, Sci, 298, 1756

Montmerle, T., Augereau, J.-C., Chaussidon, M., et al. 2006, Earth Moon Planets, 98, 39

Morbidelli, A., \& Nesvorný, D. 2020, in The Trans-Neptunian Solar System, eds. D. Prialnik, M. A. Barucci, \& L. Young, 25

Murray, C. D., \& Dermott, S. F. 1999, Solar System Dynamics (Cambridge: Cambridge University Press)

Nayakshin, S. 2010, MNRAS, 408, L36

Nayakshin, S. 2017, PASA, 34, e002

Nayakshin, S., \& Cha, S.-H. 2012, MNRAS, 423, 2104

Nesvorný, D., Vokrouhlický, D., Dones, L., et al. 2017, ApJ, 845, 27

Oort, J. H. 1950, Bull. Astron. Inst. Neth., 11, 91

Safronov, V. S. 1991, Icarus, 94, 260

Saillenfest, M., Fouchard, M., Ito, T., \& Higuchi, A. 2019, A\&A, 629, A95

Sefilian, A. A., \& Touma, J. R. 2019, AJ, 157, 59

Shankman, C., Kavelaars, J. J., Bannister, M. T., et al. 2017a, AJ, 154, 50

Shankman, C., Kavelaars, J. J., Lawler, S. M., Gladman, B. J., \& Bannister, M. T. 2017b, AJ, 153, 63

Stamatellos, D. 2015, ApJ, 810, L11

Terquem, C., \& Papaloizou, J. C. B. 2002, MNRAS, 332, L39

Trujillo, C. A. in The Trans-Neptunian Solar System, eds. D. Prialnik, M. A. Barucci, \& L. Young, 79

Trujillo, C. A., \& Sheppard, S. S. 2014, Nature, 507, 471

Von Zeipel, H. 1910, Astron. Nachr., 183, 345

Vorobyov, E. I. 2013, A\&A, 552, A129

Vorobyov, E. I., \& Basu, S. 2005, ApJ, 633, L137

Vorobyov, E. I., \& Elbakyan, V. G. 2018, A\&A, 618, A7

Vorobyov, E. I., \& Elbakyan, V. G. 2019, A\&A, 631, A1

Vorobyov, E. I., \& Pavlyuchenkov, Y. N. 2017, A\&A, 606, A5

Wahlberg Jansson, K., \& Johansen, A. 2014, A\&A, 570, A47

Weidenschilling, S. J. 1977, Ap\&SS, 51, 153

Zderic, A., Collier, A., Tiongco, M., \& Madigan, A.-M. 2020, ApJ, 895, L27

Zhu, Z., Hartmann, L., Nelson, R. P., \& Gammie, C. F. 2012, ApJ, 746, 110 


\section{Appendix A: Orbital elements of distant multiple-opposition TNOs}

Table A.1. Heliocentric orbital elements of distant multiple-opposition TNOs at epoch 2020 May 31.0.

\begin{tabular}{lccccc}
\hline \hline Object & $\begin{array}{c}a \\
(\mathrm{au})\end{array}$ & $\begin{array}{c}q \\
(\mathrm{au})\end{array}$ & $\begin{array}{c}i \\
(\mathrm{deg})\end{array}$ & $\begin{array}{c}\omega \\
(\mathrm{deg})\end{array}$ & $\begin{array}{c}\Omega \\
(\mathrm{deg})\end{array}$ \\
\hline 2000 CR105 & $216.1 \pm 0.6$ & $44.076 \pm 0.005$ & $22.8207 \pm 0.0006$ & $316.38 \pm 0.01$ & $128.3726 \pm 0.0003$ \\
2003 VB12 & $484.4 \pm 0.3$ & $76.257 \pm 0.005$ & $11.9307 \pm 0.0000$ & $311.35 \pm 0.01$ & $144.2479 \pm 0.0011$ \\
2004 VN112 & $326.7 \pm 1.9$ & $47.288 \pm 0.007$ & $25.5851 \pm 0.0003$ & $326.73 \pm 0.01$ & $65.9868 \pm 0.0005$ \\
2010 GB174 & $342.3 \pm 22.5$ & $48.645 \pm 0.310$ & $21.5983 \pm 0.0052$ & $347.13 \pm 0.36$ & $130.8929 \pm 0.0198$ \\
2012 VP113 & $261.5 \pm 1.4$ & $80.399 \pm 0.089$ & $24.1082 \pm 0.0023$ & $293.57 \pm 0.37$ & $90.6829 \pm 0.0056$ \\
2013 FT28 & $304.8 \pm 10.1$ & $43.394 \pm 0.113$ & $17.3579 \pm 0.0034$ & $40.78 \pm 0.16$ & $217.7497 \pm 0.0048$ \\
2013 GP136 & $153.9 \pm 0.2$ & $41.009 \pm 0.009$ & $33.5230 \pm 0.0006$ & $42.70 \pm 0.04$ & $210.7320 \pm 0.0001$ \\
2013 RA109 & $479.5 \pm 2.3$ & $45.977 \pm 0.015$ & $12.4053 \pm 0.0001$ & $262.79 \pm 0.15$ & $104.6721 \pm 0.0055$ \\
2013 SY99 & $728.0 \pm 25.0$ & $50.078 \pm 0.056$ & $4.2223 \pm 0.0012$ & $31.83 \pm 0.11$ & $29.5104 \pm 0.0052$ \\
2013 UT15 & $198.9 \pm 0.8$ & $44.049 \pm 0.026$ & $10.6508 \pm 0.0010$ & $251.87 \pm 0.03$ & $191.9448 \pm 0.0004$ \\
2014 SR349 & $311.9 \pm 19.9$ & $47.680 \pm 0.283$ & $17.9686 \pm 0.0017$ & $340.76 \pm 0.63$ & $34.8624 \pm 0.0149$ \\
2014 SS349 & $149.5 \pm 1.2$ & $45.290 \pm 0.060$ & $48.1325 \pm 0.0025$ & $147.62 \pm 0.14$ & $144.1310 \pm 0.0011$ \\
2015 KE172 & $132.7 \pm 0.1$ & $44.141 \pm 0.002$ & $38.3204 \pm 0.0010$ & $15.75 \pm 0.08$ & $227.5674 \pm 0.0003$ \\
2015 KG163 & $805.0 \pm 5.9$ & $40.493 \pm 0.008$ & $13.9822 \pm 0.0012$ & $32.29 \pm 0.10$ & $219.0906 \pm 0.0017$ \\
2015 KH163 & $157.9 \pm 0.6$ & $39.956 \pm 0.026$ & $27.0854 \pm 0.0014$ & $230.58 \pm 0.05$ & $67.5927 \pm 0.0006$ \\
2015 RX245 & $429.1 \pm 36.3$ & $45.635 \pm 0.230$ & $12.1244 \pm 0.0028$ & $64.91 \pm 0.30$ & $8.5925 \pm 0.0002$ \\
2015 TG387 & $1085.5 \pm 111.5$ & $65.160 \pm 0.212$ & $11.6545 \pm 0.0006$ & $117.78 \pm 0.32$ & $300.7795 \pm 0.0072$ \\
2018 VM35 & $251.9 \pm 64.0$ & $44.962 \pm 1.282$ & $8.4808 \pm 0.0034$ & $302.91 \pm 2.68$ & $192.4070 \pm 0.0584$ \\
\hline
\end{tabular}

Table A.1 gives the heliocentric orbital elements and their standard deviations for the TNOs with $a>100$ au used in Sect. 2. These values were taken from the Jet Propulsion Laboratory website $^{2}$ on September 2, 2020.

\section{Appendix B: Dynamical evolution of the studied clumps}

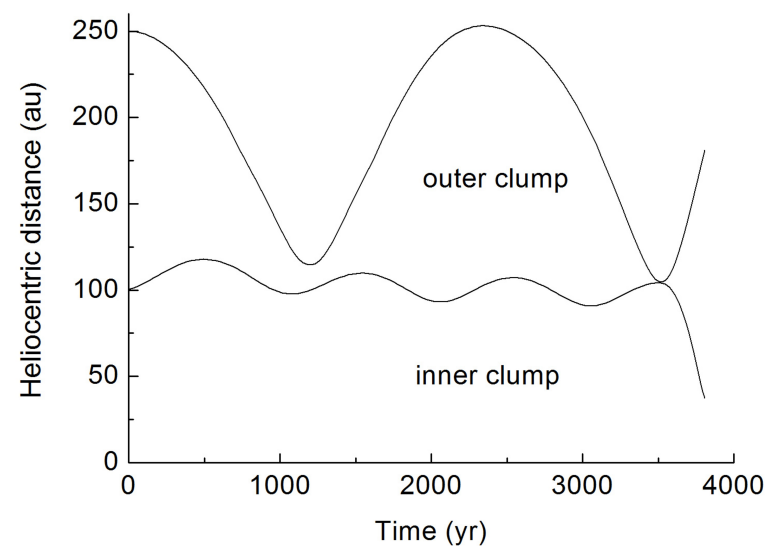

Fig. B.1. Heliocentric distances of two clumps.

Figure B.1 shows the heliocentric distances of two clumps as a function of time.

\footnotetext{
2 https://ssd.jpl.nasa.gov/sbdb.cgi
}

\section{Appendix C: Orbital distribution of particles before a close encounter}

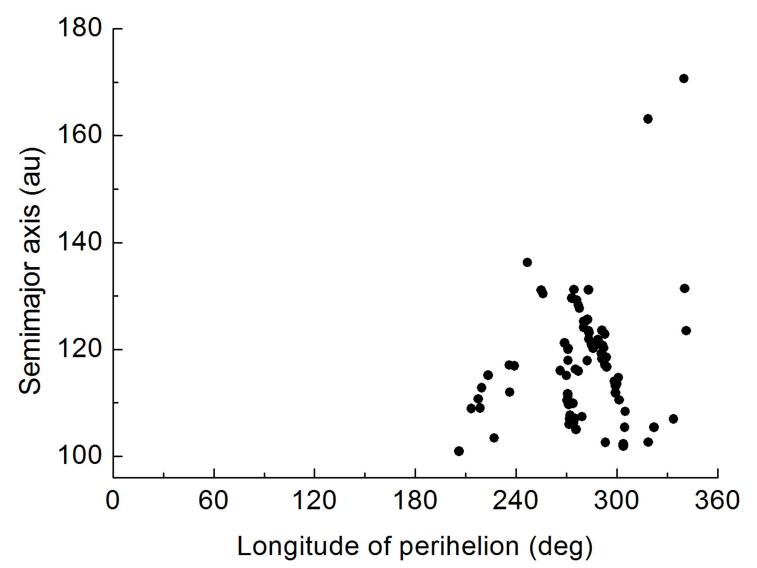

Fig. C.1. Distribution of longitudes of perihelion and semimajor axes for simulated particles with $40<q<80$ au and $a>100$ au after the first stage.

After 2300 years of evolution, the outer clump reaches the orbit with $a=180 \mathrm{au}$ and $q=107 \mathrm{au}$, and the inner clump moves in the orbit with $a=100$ au and the aphelion distance $Q=107 \mathrm{au}$. Figure C. 1 shows the distribution of longitudes of perihelion and semimajor axes for particles with $40<q<80$ au and $a>100$ au (the observable region of distant TNOs) at this time. Only particles located outside the Hill region are included. All these particles originate in the Hill region of the outer clump. The inner clump does not contribute to the observable region at this stage. The longitudes of perihelion of the particles are concentrated near the value of $\pi$ of the outer clump. 